\title{
Auslander Modules and Quasi-Homogeneity of Local Rings
}

\author{
By \\ Yuji Yoshino* and Kiriko Kato**
}

\section{§0. Introduction}

Let $(R, \mathrm{~m}, k)$ be a normal local domain of dimension two and assume that $k$ is an algebraically closed field of characteristic zero. Let us denote $K_{R}$ the canonical module of $R$. Then from the definition of $K_{R}$, there are isomorphisms:

$$
\operatorname{Ex} t_{R}^{1}\left(\mathrm{~m}, K_{R}\right) \cong \operatorname{Ex} t_{R}^{2}\left(k, K_{R}\right) \cong k,
$$

which involves the unique existence of the nonsplit exact sequence:

$$
0 \rightarrow K_{R} \rightarrow A \rightarrow \mathrm{m} \rightarrow 0
$$

The $R$-module $A$ which appears in the sequence is also uniquely determined except for isomorphisms, and is called the Auslander module of $R$. (For more details, see [13; Chapter 11].) In this paper, it is of our main interest how the Auslander module is related to the quasi-homogeneity of a local ring. Here recall that $R$ is said to be quasi-homogeneous if it is the completion of a positively graded ring, or equivalently if it has an Euler derivation, i.e. a $k$-derivation $\Delta$ with the property that

$$
\Delta\left(X_{i}\right)=d_{i} X_{i} \quad(1 \leq i \leq n)
$$

for some system of generators $X_{1}, X_{2}, \cdots, X_{n}$ of $\mathrm{m}$ and positive integers $d_{1}, d_{2}, \cdots, d_{n}$.

Now to fix our attention onto a ring of hypersurface singularity, we put $R=P / f P$ where $P=k[[X, Y, Z]]$ is the formal power series ring and

Communicated by K. Saito, May 13, 1993. Revised February 2, 1994.

1991 Mathematics Subject Classifications: 13C14, 13D03, 13N05, $14 J 17$.

* Division of Mathematics, Dept. of Fundamental Science, Faculty of Integrated Human Studies, Kyoto University, Kyoto 606-01, Japan.

** Research Institute for Mathematical Sciences, Kyoto University, Kyoto 606-01, Japan. 
$f \in \mathrm{m}_{P}=(X, Y, Z) P$, and assume that $R$ is non-regular to avoid the trivial case.

First consider the case that $R$ is quasi-homogeneous, and we shall make it clear how Auslander module is represented. Denoting the universally finite module of differentials of $R$ by $D=D_{k}(R)$, we may take an $R$-epimorphism $\delta: D \rightarrow \mathrm{m}$ corresponding to the Euler derivation and obtain the following:

$$
\begin{aligned}
& \operatorname{Ker}\left(\delta^{* *}\right) \cong R, \\
& \operatorname{Im}\left(\delta^{* *}\right)=\mathrm{m} .
\end{aligned}
$$

In fact, $(0.1)$ is obtained from the isomorphisms $\operatorname{Ker}\left(\delta^{* *}\right) \cong\left(\bigwedge^{2} D\right)^{* *} \cong K_{R} \cong R$. In order to show $(0.2)$, assume the contrary. Then it would be $\operatorname{Im}\left(\delta^{* *}\right)=R$, and this together with $(0.1)$ yeilds that $D^{* *} \cong R^{2}$. Thus by the Zariski-Lipman conjecture established for hypersurfaces by Scheja-Storch [9], $R$ would be a regular local ring, which contradicts the hypothesis.

It hence follows from (0.1) and (0.2) that there is a non-split exact sequence:

$$
0 \rightarrow R \rightarrow D^{* *} \rightarrow \mathrm{m} \rightarrow 0 .
$$

Therefore the Auslander module is isomorphic to $D^{* *}$ in this case.

Martsinkovsky ([6]) have conjectured the converse of this claim:

Conjecture (0.3). The following conditions would be equivalent for $R$ :

(0.3.1) $R$ is quasi-homogeneous.

(0.3.2) The Auslander module is isomorphic to $D^{* *}$.

We remark that the above argument shows the condition (0.3.2) is equivalent to:

(0.3.3) There exists an epimorphism $D^{* *} \rightarrow m$.

Meanwhile we must also remark that there are some known equivalent conditions for (0.3.1):

(0.3.4) $f \in\left(f_{X}, f_{Y}, f_{Z}\right) P$. (cf. Saito [8])

(0.3.5) There exists an epimorphism $D \rightarrow m$. (cf. Scheja-Wiebe [11])

Therefore the Martsinkovsky's conjecture is reduced to the following: 
Problem (0.4). When there is an R-epimorphism $D^{* *} \rightarrow \mathrm{m}$, is there also an epimorphism $D \rightarrow m$ ? (See also [6; Prop. 2.2].)

In this problem it is intrinsically required to get a property of $D$ from that of $D^{*}=\operatorname{Der}_{k}(R, R)$, hence in a sense there seems to have similar difficulty as in proving the Zariski-Lipman conjecture.

We exhibit some types of hypersurfaces for which the conjecture is to have an affirmative answer.

(0.5). If $f$ is one of the following equations, then the conjecture (0.3) holds true for $R=P / f P$ :

$$
f=X^{n}+g(Y, Z)(\text { cf. Martsinkovsky [6]) }
$$

(0.5.2) $f=X^{p}+Y^{q}+Z^{r}+X Y Z$ (cf. Behnke [1] or Yoshino-Kawamoto [14])

(0.5.3) $f=g+H(g)$, where $g=X^{p}+Y^{q}+Z^{r}$ and $H(g)$ denotes the Hessian of $g$.

In this paper we shall show these are really the cases for which the conjecture is true. For (0.5.1), we give another proof which seems to be simpler than the Martsinkovsky's original one. The cases for (0.5.2) and (0.5.3) will be treated as only a matter of calculation. Yet for the rings of hypersurfaces in general form, we have to say that the problem remains unsolved.

Martsinkovsky's latest work [7] was regarded as proving this conjecture for all the rings of hypersurface, but the proof contains a certain serious gap and cannot be an answer to the problem. In [7], he tried to prove it with the help of a nonminimal free resolution over $R$, what we call an Eisenbud resolution, of $R / \overline{j(f)}$, where $\overline{j(f)}$ denotes an $R$-ideal generated by all the partials of $f$ :

$$
\cdots \longrightarrow R^{n+3} \oplus R \stackrel{\phi_{3}}{\longrightarrow} R^{n} \oplus R^{4} \stackrel{\phi_{2}}{\longrightarrow} R^{n+3} \oplus R \stackrel{\phi_{1}}{\longrightarrow} R^{4} \stackrel{\phi_{0}}{\longrightarrow} R
$$

He focused on the structure of $\phi_{3}$, with the assumption of the conjecture, whose cokernel is to be isomorphic to the first syzygy module $\Omega^{1} A$ of $A$ up to a free direct summand. While the minimal presentation matrix of $\Omega^{1} A$ can be explicitly computed. It is true that, omitting a free summand from $\operatorname{Coker}\left(\phi_{3}\right)$, $\Omega^{1} A$ is isomorphic to the cokernel of a map $\tilde{\tau}: R^{4} \rightarrow R^{n+3}$, where the matrix $\tilde{\tau}$ has the property that the last three entries in each column are the coefficients of relations over $R$ among the partials of $f$. On the other hand, there certainly exists a map $\tau: R^{4} \rightarrow R^{4}$ whose cokernel is isomorphic to $\Omega^{1} A$. In [7: page 
553, lines 13-17], he claims with no explanation, that also for this $\tau$, the matrix has the same property as $\tilde{\tau}$. But it is not only unguaranteed, but also an essential part to prove the conjecture. (If it were true, then our result (3.14) or a theorem of Herzog [4] would have led us to a proof of the conjecture.)

We attempt in this paper to solve the conjecture $(0.3)$ mainly in a vein of commutative algebra. The homological algebra on local rings is expected to play a central role in this problem, since, as mentioned above, it has a resemblance to the Zariski-Lipman conjecture. From this viewpoint, we make a translation of the conditions such as the existence of $R$-epimorphism $D^{* *} \rightarrow \mathrm{m}$, or the quasi-homogeneity of $R$, into homological language. In consequence, we shall get several sufficient conditions for the conjecture $(0.3)$ being valid.

In $\S 1$ we will exhibit, in a quite general form, those of our results which can be discussed purely homologically and which is independent of the properties of the module of differentials. More precisely, we show some calculations to get the homologies of a kind of extended Koszul complex. This will be done only by routine computation, but we include it here, because it has never appeared in other articles.

The subsequent section $\S 2$ is devoted to rewrite the results of $\S 1$ in the form convenient for later usage.

The main body of the paper is $\S 3$, in which we will show that the structure of the Koszul homology with respect to the sequence $\nabla f=\left(f_{x}, f_{y}, f_{z}\right)$ gives an important information for the assumption or the conclusion of the problem (0.4). Actually, under the assumption of (0.4), we can find an element $a=\left(a_{1}, a_{2}, a_{3}\right)$ in $R^{3}$ which makes $\nabla f$ a nontrivial socle of $H_{1}(a)$ (cf. (3.4)). This sequence $a$ looks a key to the problem. And we show several properties of $a$ in $\S 3$. In fact, we can show that if gives an element of $m H_{1}(\nabla f)$, then $R$ is quasi-homogeneous, as desired (cf. (3.12)). Furthermore, we also give, in Theorem (3.15), some necessary conditions for the condition (0.3.2) to hold, by which we can verify the conjecture for a given equation only through calculating $H_{1}(\nabla f)$.

Making use of these results, in $\S 4$ we shall actually verify the conjecture for the equations in (0.5).

\section{$\S 1$. Homologies of Some Complexes of Koszul Type}

Throughout this section, let $(R, m, k)$ be a Cohen-Macaulay local ring of dimension $d$, where $d$ is assumed to be not less than two. And let $F$ be a free module over $R$ of rank $d+1$ generated by $\left\{\boldsymbol{e}_{i} \mid i=1,2, \cdots, d+1\right\}$, so that 
$F=\sum_{i=1}^{d+1} R e_{i} \cong R^{d+1}$. We take two elements $\boldsymbol{a}=\left(a_{1}, a_{2}, \cdots, a_{d+1}\right), \boldsymbol{b}=\left(b_{1}, b_{2}, \cdots\right.$, $\left.b_{d+1}\right)$ in $F$ satisfying the properties that:

(1.1.1) Both $\mathfrak{a}=\sum_{i=1}^{d+1} a_{i} R$ and $\mathfrak{b}=\sum_{i=1}^{d+1} b_{i} R$ are m-primary ideals, and

(1.1.2) As an element of $R$, the inner product $\boldsymbol{a} \cdot \boldsymbol{b}$ vanishes, i.e. $\sum_{i=1}^{d+1} a_{i} b_{i}=0$.

Then, for each $n(0 \leq n \leq d+1)$, we can consider two kinds of maps:

$$
\Delta_{a}^{-}: \bigwedge^{n} F \longrightarrow \bigwedge^{n-1} F \text { and } \Delta_{b}^{+}: \bigwedge^{n} F \longrightarrow \bigwedge^{n+1} F
$$

defined by the following rules:

$$
\begin{aligned}
\Delta_{\boldsymbol{a}}^{-}\left(\boldsymbol{e}_{i_{1}} \wedge \boldsymbol{e}_{i_{2}} \wedge \cdots \wedge \boldsymbol{e}_{i_{n}}\right) & =\sum_{j=1}^{n}(-1)^{j+1} a_{i_{j}} \boldsymbol{e}_{i_{1}} \wedge \cdots \wedge \hat{\boldsymbol{e}}_{i_{j}} \wedge \cdots \wedge \boldsymbol{e}_{i_{n}} \\
\Delta_{\boldsymbol{b}}^{+}(\omega) & =\boldsymbol{b} \wedge \omega .
\end{aligned}
$$

The complex

$$
0 \longrightarrow \bigwedge^{d+1} F \stackrel{\Delta_{a}^{-}}{\longrightarrow} \bigwedge^{d} F \stackrel{\Delta_{a}^{-}}{\longrightarrow} \ldots \rightarrow \bigwedge^{1} F \stackrel{\Delta_{a}^{-}}{\longrightarrow} \bigwedge^{0} F \longrightarrow 0
$$

is the Koszul complex relative to the sequence $a$, whose $n$-th homology is denoted by $H_{n}(\boldsymbol{a})$ as usual. Similarly $H^{n}(\boldsymbol{b})$ is the $n$-th cohomology of the Koszul co-complex:

$$
0 \longrightarrow \bigwedge^{0} F \stackrel{\Delta_{b}^{+}}{\rightarrow} \bigwedge^{1} F \stackrel{\Delta_{b}^{+}}{\longrightarrow} \cdots \longrightarrow \bigwedge^{d} F \stackrel{\Delta_{b}^{+}}{\longrightarrow} \bigwedge^{d+1} F \longrightarrow 0 .
$$

Note that $H_{n}(\boldsymbol{a})=0(n \neq 0,1)$ and $H^{n}(\boldsymbol{b})=0(n \neq d, d+1)$, since the ideals $a$ and $\mathrm{b}$ are m-primary and thus have grade $d$. See [4; Prop. 1.4.4] for example.

From the definition, the equation $\Delta_{a}^{-} \Delta_{b}^{+}+\Delta_{b}^{+} \Delta_{a}^{-}=0$ is easily seen to hold. And this enables us to define the double complex $G_{. .}$as follows

$$
G_{p q}= \begin{cases}\bigwedge^{p-q} F & \text { if } p \geq q \geq 0 \\ 0 & \text { otherwise }\end{cases}
$$

with differential maps $\Delta_{a}^{-}$and $\Delta_{b}^{+}$: 


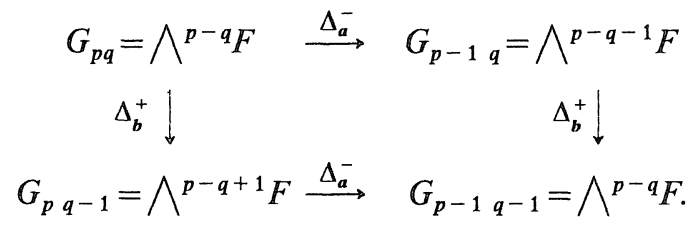

We now define the complex $M_{\bullet}$ that is our main concern in this section.

Definition (1.2). Let $M$ be the module defined by the following exact sequence:

$$
0 \longrightarrow R \stackrel{\Delta_{b}^{+}}{\longrightarrow} F \longrightarrow M \longrightarrow 0
$$

The complex $M_{\circ}$ is defined as

$$
0 \rightarrow \bigwedge^{d+1} M \stackrel{\delta_{a}}{\rightarrow} \bigwedge^{d} M \stackrel{\delta_{a}}{\rightarrow} \cdots \rightarrow \bigwedge^{1} M \stackrel{\delta_{a}}{\rightarrow} \bigwedge^{0} M \rightarrow 0
$$

where $\delta_{a}$ is given by a similar rule as $\Delta_{a}^{-}$:

$$
\delta_{a}\left(m_{i_{1}} \wedge m_{i_{2}} \wedge \cdots \wedge m_{i_{n}}\right)=\sum_{j=1}^{n}(-1)^{j+1} a_{i_{j}} m_{i_{1}} \wedge \cdots \wedge \hat{m}_{i_{j}} \wedge \cdots \wedge m_{i_{n}}
$$

Remark (1.3). We can easily verify that $M_{0}$ is well-defined as a complex. It actually goes through as follows:

First consider the $R$-modules $M_{n}(n \geq 0)$ defined by the exact sequences:

$$
\bigwedge^{n-1} F \stackrel{\Delta_{b}^{+}}{\longrightarrow} \bigwedge^{n} F \longrightarrow M_{n} \longrightarrow 0 .
$$

Then there are natural isomorphisms of $R$-modules $M_{n} \cong \wedge^{n} M$ for each integer $n$, and under these isomorphisms, the maps $\delta_{a}$ are the ones induced by $\Delta_{a}^{-}$, that is, so as to make the diagram below commutative:

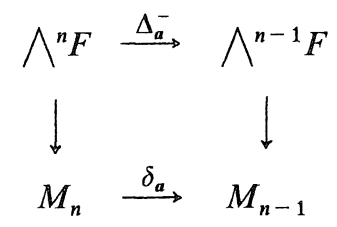


Definition (1.4). In a quite similar way as in (1.3), we can define $R$-modules $N_{n}(n \geq 0)$ by the exact sequences:

$$
0 \rightarrow N_{n} \rightarrow \bigwedge^{n+1} F \stackrel{\Delta_{b}^{+}}{\rightarrow} \bigwedge^{n+2} F
$$

And the complex $N_{0}$ is defined as:

$$
0 \rightarrow N_{d+1} \stackrel{\varepsilon_{a}}{\rightarrow} N_{d} \stackrel{\varepsilon_{a}}{\rightarrow} \cdots \rightarrow N_{1} \stackrel{\varepsilon_{a}}{\rightarrow} N_{0} \rightarrow 0
$$

where the maps $\varepsilon_{\boldsymbol{a}}$ are the induced ones by $\Delta_{\boldsymbol{a}}^{-}$with the commutative diagram:

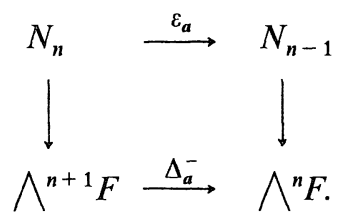

Remark (1.5). Note that the modules $H^{n}(b)$ are of finite length. Thus if $\mathfrak{p}$ is a prime ideal of $R$ with $\mathfrak{p} \neq \mathfrak{m}$, then $H^{n}(b)_{\mathfrak{p}}=0$, hence $\left(N_{n}\right)_{\mathfrak{p}}$ and $\left(M_{n}\right)_{\mathfrak{p}}$ are free modules over $R_{\mathfrak{p}}$ for all $n$.

Now we remark that each $N_{n}$ in the complex $N_{0}$ is a reflexive $R$-module. In fact, consider the canonical map $g_{n}: N_{n} \rightarrow\left(N_{n}\right)^{* *}$ for each $n$, where ( $)^{*}$ denotes the $R$-dual $\operatorname{Hom}_{R}(, R)$, and we can show from the above that $\operatorname{Ker}\left(g_{n}\right)$ and $\operatorname{Coker}\left(g_{n}\right)$ are of finite length. But since we have assumed that $d \geq 2$ and since $N_{n}$ is a second syzygy module, we have $\operatorname{depth}\left(N_{n}\right) \geq 2$ and $\operatorname{depth}\left(\left(N_{n}\right)^{* *}\right) \geq 2$. It therefore follows that $g_{n}$ is an isomorphism.

Note also that there is a natural map $\gamma_{n}: M_{n} \rightarrow N_{n}$ for any $n$, which is in fact induced by the map $\Delta_{b}^{+}: \wedge^{n} F \rightarrow \wedge^{n+1} F$.

A simple observation leads us to the following:

\section{Lemma (1.6).}

(1.6.1) For each $n$, there is an exact sequence:

$$
0 \longrightarrow H^{n}(\boldsymbol{b}) \longrightarrow M_{n} \stackrel{\gamma_{n}}{\rightarrow} N_{n} \rightarrow H^{n+1}(\boldsymbol{b}) \rightarrow 0
$$

(1.6.2) There are the maps $d_{\boldsymbol{a}}: H^{n+1}(\boldsymbol{b}) \rightarrow H^{n}(\boldsymbol{b})$ naturally induced from $\Delta_{\boldsymbol{a}}^{-}$, and they make the following diagram commutative: 


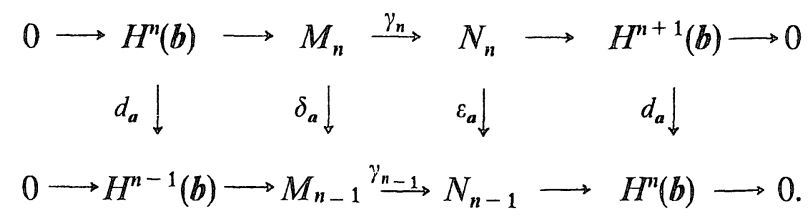

Similarly the natural maps $d_{b}: H_{n}(a) \rightarrow H_{n+1}(a)(0 \leq n \leq d)$ are induced from $\Delta_{b}^{+}$.

Proof. The statement (1.6.1) is straightforward from the definition. To show (1.6.2), note that the complex $G_{p}$ is the shifting of the Koszul co-complex relative to $b$ and that $\Delta_{a}^{-}: G_{p \circ} \rightarrow G_{p-1}$ 。 gives a chain map, for every $p$. Thus (1.6.2) follows by taking the homology of this chain map and by noting its naturality.

Corollary (1.7). There is a natural isomorphism of complexes:

$$
\left(M_{0}\right)^{* *} \cong N_{0}
$$

Proof. Note that the modules $H^{n}(b)$ are of finite length, hence that $H^{n}(\boldsymbol{b})^{*}=0$ and $\operatorname{Ext}_{R}^{1}\left(H^{n}(\boldsymbol{b}), R\right)=0$. It thus follows from (1.6.1) that $\gamma_{n}^{*}$ is an isomorphism for each $n$. Therefore, since $N_{n}$ is a reflexive module by (1.5), we have an isomorphism $N_{n} \cong\left(M_{n}\right)^{* *} \cong\left(\wedge^{n} M\right)^{* *}$ for each $n$. Furthermore we can see from (1.6.2) that under these isomorphisms, $\varepsilon_{a}=\delta_{a}^{* *}$.

The next lemma will be necessary in later sections.

Lemma (1.8). The following is exact:

$$
0 \longrightarrow\left(\bigwedge^{d} M\right)^{* *} \stackrel{\delta_{a}^{* *}}{\longrightarrow}\left(\bigwedge^{d-1} M\right)^{* *} \stackrel{\delta_{a}^{* *}}{\longrightarrow}\left(\bigwedge^{d-2} M\right)^{* *}
$$

Proof. It suffices from (1.7) to prove that the following sequence is exact:

$$
0 \longrightarrow N_{d} \stackrel{\varepsilon_{a}}{\longrightarrow} N_{d-1} \stackrel{\varepsilon_{a}}{\longrightarrow} N_{d-2}
$$

But this is clear since there is a commutative diagram with exact columns and since the second and the third rows of this diagram are exact: 


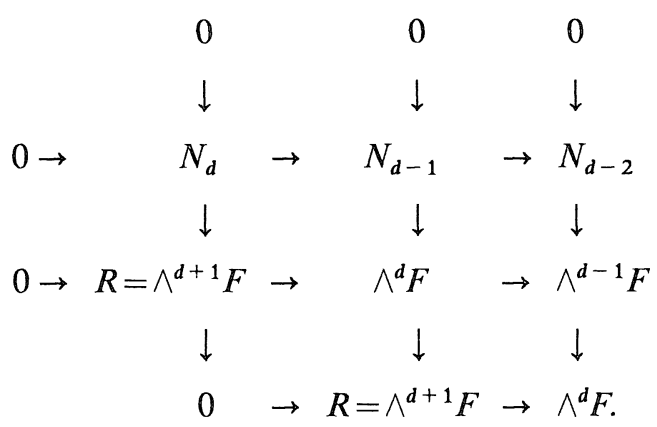

(Note that the second and the third rows are exact, because a has grade greater than one.)

Summing up the above results, we have the following:

Corollary (1.9). There is a commutative diagram:

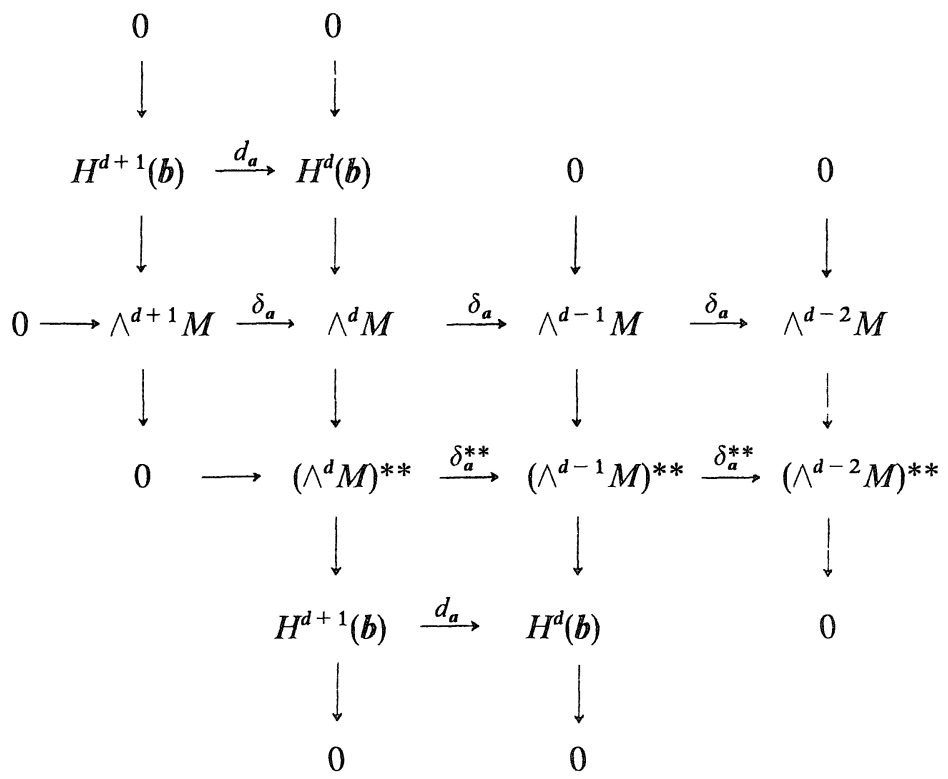

where the columns and the third row are exact.

Remark (1.10). The maps $d_{a}: H^{n+1}(b) \rightarrow H^{n}(b)$ (resp. $d_{b}: H_{n}(a) \rightarrow H_{n+1}(a)$ ) are non-trivial only for a single value $n=d$ (resp. $n=0$ ). Therefore, with no fear of confusion, we adopt the notation $\operatorname{Ker}\left(d_{\boldsymbol{a}}\right)$ or $\operatorname{Coker}\left(d_{\boldsymbol{a}}\right)$ as for the kernel 
or the cokernel of the map $d_{a}: H^{d+1}(b) \rightarrow H^{d}(b)$. Likewise $\operatorname{Ker}\left(d_{b}\right)$ and $\operatorname{Coker}\left(d_{b}\right)$ are the kernel and the cokernel of $d_{b}: H_{0}(a) \rightarrow H_{1}(a)$ respectively.

Now we give our main result concerning the homologies of the complex $M_{0}$. In order to state the theorem we denote the homology of $M_{\circ}$ by $H_{\circ}$, i.e. $H_{n}=H_{n}\left(M_{0}\right)$.

Theorem (1.11).

(1.11.1) [periodicity] There are isomorphisms of $R$-modules:

$$
\begin{aligned}
& \mathbb{H}_{0} \cong H_{0}(a), \\
& \mathbb{H}_{1} \cong \mathbb{H}_{3} \cong \cdots \cong \mathbb{H}_{2 n+1}\left(=\mathbb{H}_{\text {odd }}\right) \quad \text { for } 1 \leq 2 n+1 \leq d+1, \text { and } \\
& \mathbb{H}_{2} \cong \mathbb{H}_{4} \cong \cdots \cong \mathbb{H}_{2 n}\left(=\mathbb{H}_{\text {even }}\right) \quad \text { for } 1 \leq 2 n \leq d+1 .
\end{aligned}
$$

(1.11.2) If $d$ is even, then we have:

$$
\mathbb{H}_{e v e n} \cong \operatorname{Ker}\left(d_{b}\right) \cong \operatorname{Coker}\left(d_{a}\right), \quad \mathbb{H}_{o d d} \cong \operatorname{Coker}\left(d_{b}\right) \cong \operatorname{Ker}\left(d_{a}\right)
$$

In contrast, when $d$ is odd, there are isomorphisms:

$$
\mathbb{H}_{e v e n} \cong \operatorname{Ker}\left(d_{b}\right) \cong \operatorname{Ker}\left(d_{a}\right), \quad \mathbb{H}_{\text {odd }} \cong \operatorname{Coker}\left(d_{b}\right) \cong \operatorname{Coker}\left(d_{\boldsymbol{a}}\right) .
$$

(1.11.3) [rigidity] If there is an integer $i$ with $0<i \leq d+1$ such that $\mathbb{H}_{i}=0$, then the complex $M$ 。 is acyclic.

Proof. To show the theorem we have only to calculate the spectral sequence associated with the double complex $G_{\text {o }}$.

For the first it is carried out with taking the row homologies to get the $\mathbb{E}^{1}$ terms:

$$
E_{p q}^{1}=H_{p}\left(G_{\circ q}\right)= \begin{cases}H_{0}(a) & (q=p \geq 0) \\ H_{1}(a) & (q=p-1 \geq 0) \\ 0 & \text { (otherwise) }\end{cases}
$$

and note that the maps $E_{p p}^{1} \rightarrow E_{p p-1}^{1}$ are nothing but $d_{b}$. Thus the $E^{2}$ terms are: 


$$
E_{p q}^{2}=H_{q}\left(E_{p}^{1} \text { o }\right)= \begin{cases}H_{0}(a) & (q=p=0) \\ \operatorname{Ker}\left(d_{b}\right) & (q=p>0) \\ \operatorname{Coker}\left(d_{b}\right) & (q=p-1>0) \\ 0 & \text { (otherwise) }\end{cases}
$$

Therefore the homology of the total complex of $G_{\bullet}$. is given as follows:

$$
H_{n}\left(G_{\bullet}\right)= \begin{cases}H_{0}(\boldsymbol{a}) & (n=0) \\ \operatorname{Coker}\left(d_{\boldsymbol{b}}\right) & (n: \text { positive and odd }) \\ \operatorname{Ker}\left(d_{\boldsymbol{b}}\right) & (n: \text { positive and even })\end{cases}
$$

On the other hand, we can calculate this homology by starting with column homologies. We write in this case, ' $E^{1}$ as the $E^{1}$ term, and so on.

$$
{ }^{\prime} E_{p q}^{1}=H_{q}\left(G_{p o}\right)= \begin{cases}\wedge^{p} M & (q=0,0 \leq p \leq d+1) \\ H^{d}(\boldsymbol{b}) & (q=p-d>0) \\ H^{d+1}(\boldsymbol{b}) & (q=p-d-1>0) \\ 0 & \text { (otherwise) }\end{cases}
$$

Thus,

$$
' E_{p q}^{2}=H_{p}\left(E_{\bullet q}^{1}\right)= \begin{cases}H_{p} & (q=0,0 \leq p \leq d+1) \\ \operatorname{Coker}\left(d_{a}\right) & (q=p-d>0) \\ \operatorname{Ker}\left(d_{a}\right) & (q=p-d-1>0) \\ 0 & \text { (otherwise) }\end{cases}
$$

Therefore we finally obtain another description of the homologies of the total complex:

$$
H_{n}\left(G_{\bullet}\right)= \begin{cases}\boldsymbol{H}_{n} & (0 \leq n \leq d+1) \\ \operatorname{Coker}\left(d_{\boldsymbol{a}}\right) & (n>d+1 \text { and } n-d \text { is even }) \\ \operatorname{Ker}\left(d_{\boldsymbol{a}}\right) & (n>d+1 \text { and } n-d \text { is odd })\end{cases}
$$

Now the theorem is obtained by comparing the above two descriptions of $H_{n}\left(G_{\bullet \bullet}\right)$. (For the rigidity, we have only to notice that $\boldsymbol{H}_{\text {even }}$ and $\boldsymbol{H}_{\text {odd }}$ have the same length by (1.11.2).)

\section{§2. The Case of Dimension Two}

In this section we apply the results of the previous section to the case $d=2$. Thus we assume that $R$ is a Cohen-Macaulay local ring of dimension 
two with maximal ideal $m$. Recalling the notation in $\S 1$, we have:

Notation (2.1). Let $F$ be a free module over $R$ of rank 3: $F=R e_{1} \oplus R e_{2}$ $\oplus R e_{3} \cong R^{3}$, and let $a=\left(a_{1}, a_{2}, a_{3}\right)$ and $b=\left(b_{1}, b_{2}, b_{3}\right)$ be two elements of $F$ satisfying the conditions:

(2.1.1) The ideals $\mathfrak{a}=\left(a_{1}, a_{2}, a_{3}\right) R$ and $\mathfrak{b}=\left(b_{1}, b_{2}, b_{3}\right) R$ are both m-primary ideals of $R$, and

$$
a \cdot b=a_{1} b_{1}+a_{2} b_{2}+a_{3} b_{3}=0
$$

Remark (2.2). We remark that there is an isomorphism $\wedge^{2} F \cong F$ by the maps: $\mathfrak{e}_{2} \wedge \mathfrak{e}_{3} \mapsto \mathfrak{e}_{1}, e_{3} \wedge e_{1} \mapsto e_{2}$ and $e_{1} \wedge e_{2} \mapsto e_{3}$. Likewise $\wedge^{3} F$ is isomorphic to $R$ via $e_{1} \wedge e_{2} \wedge e_{3} \mapsto 1$.

In the rest of the paper, we identify these modules under the isomorphisms. Thus notice that the Koszul complex relative to the sequence $b$ is described as:

$$
0 \longrightarrow R \stackrel{b}{\longrightarrow} F \stackrel{b \times}{\longrightarrow} F \stackrel{b \bullet}{\longrightarrow} R \longrightarrow 0
$$

where $b \times($ resp. $b \circ)$ denotes the exterior (resp. inner) product between elements of $F$, i.e.

$$
\boldsymbol{b} \times \boldsymbol{x}=\left(\left|\begin{array}{ll}
b_{2} & b_{3} \\
x_{2} & x_{3}
\end{array}\right|,\left|\begin{array}{ll}
b_{3} & b_{1} \\
x_{3} & x_{1}
\end{array}\right|,\left|\begin{array}{ll}
b_{1} & b_{2} \\
x_{1} & x_{2}
\end{array}\right|\right)
$$

and $\boldsymbol{b} \circ \boldsymbol{x}=b_{1} x_{1}+b_{2} x_{2}+b_{3} x_{3}$ for any $\boldsymbol{x}=\left(x_{1}, x_{2}, x_{3}\right) \in F$. Note that as $R$-linear maps, they are respectively represented by the matrices:

$$
\mathbb{K}_{\boldsymbol{b}}=\left(\begin{array}{ccc}
0 & b_{3} & -b_{2} \\
-b_{3} & 0 & b_{1} \\
b_{2} & -b_{1} & 0
\end{array}\right) \quad \text { and } \quad{ }^{t} \boldsymbol{b}=\left(\begin{array}{c}
b_{1} \\
b_{2} \\
b_{3}
\end{array}\right) \text {. }
$$

Let us make a remark that is an easy exercise of linear algebra.

Remark (2.3). Given three elements $x, y$ and $z$ in $F$ with the condition $x \circ y=0$, we have $y \times(x \times z)=(z \circ y) x$. In particular, $b \times(a \times z)=(z \circ b) a$ for any $z \in F$. 
This property yields the following lemma that is essentially contained in a theorem of Bruns [2]. See also Yoshino [12].

Lemma (2.4). Let $\boldsymbol{x}$ be an element of $F$. Adding to the assumption (2.1), we assume that $\boldsymbol{x} \cdot b=0$. Then $a \times x$ belongs to the subinodule $R b$ of $F$.

Proof. It follows from (2.3) and the assumption that $b \times(a \times x)=(x \cdot b) a=0$. Therefore $a \times x$ belongs to the kernel of the map $b \times$, which coincides with $R b$. (This last holds because $\mathfrak{b}$ has grade two, hence the complex $(2.2 .1)$ is exact for the first two terms.)

Remark (2.5). For an element $c$ to $F$, we denote

$$
\Omega(c)=\{x \in F \mid x \circ c=0\},
$$

which is sometimes called the relation module of $c$. By the above lemma, if $a, b \in F$ are as in (2.1), then we can define an $R$-linear map $\Psi_{a}: \Omega(b) \rightarrow R$ by the equation:

$$
a \times x=\Psi_{a}(x) b \quad \text { for any } x \in \Omega(b) .
$$

Note from (2.3) that $\Psi_{\boldsymbol{a}}(\boldsymbol{b} \times \boldsymbol{y})=\boldsymbol{a} \cdot \boldsymbol{y}$ for any $\boldsymbol{y} \in F$.

The following lemma will be useful for later discussion.

Lemma (2.6). Employing the same notation as in $\S 1$, we have an isomorphism $M^{* *} \cong \Omega(b)$ and a commutative diagram:

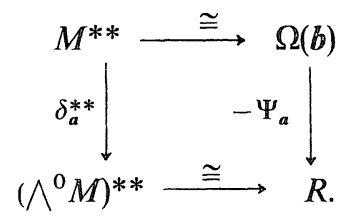

In particular, the images of $\delta_{\boldsymbol{a}}^{* *}$ and $\Psi_{\boldsymbol{a}}$ coincide with each other.

Proof. We recall from (1.7) that

$$
M^{* *} \cong N=\operatorname{Ker}\left(\bigwedge^{2} F \stackrel{\Delta_{b}^{+}}{\longrightarrow} \bigwedge^{3} F\right)
$$


and $\delta_{a}^{* *}$ is the restriction of $\Delta_{a}^{-}$on $N$, which is denoted by $\varepsilon_{a}$ in $\S 1$. However note that, under the identification as in (2.2), the map $\wedge^{2} F \stackrel{\Delta_{b}^{+}}{\rightarrow} \wedge^{3} F$ is just $F \stackrel{b \bullet}{\rightarrow} R$, therefore $N$, hence $M^{* *}$, is isomorphic to $\Omega(b)$, We should remark that an element $\boldsymbol{x}=\left(x_{1}, x_{2}, x_{3}\right) \in \Omega(b)$ corresponds to $\boldsymbol{x}^{\prime}=x_{1} \boldsymbol{e}_{2} \wedge \boldsymbol{e}_{3}+x_{2} \boldsymbol{e}_{3} \wedge \boldsymbol{e}_{1}+x_{3} \boldsymbol{e}_{1} \wedge \boldsymbol{e}_{2}$ in $N$. Thus, by definition,

$$
\begin{aligned}
\varepsilon_{a}\left(x^{\prime}\right) & =x_{1}\left(a_{2} e_{3}-a_{3} e_{2}\right)+x_{2}\left(a_{3} e_{1}-a_{1} e_{3}\right)+x_{3}\left(a_{1} e_{2}-a_{2} e_{1}\right) \\
& =-a \times x \\
& =-\Psi_{a}(x) b
\end{aligned}
$$

Since we identify $\left(\wedge^{0} M\right)^{* *} \cong N_{0}=R \boldsymbol{b}$ with $R$, the desired result follows.

Remark (2.7). We denote by $K(\boldsymbol{b})$ the image of the map $b \times: F \rightarrow F$. Note that $K(\boldsymbol{b})$ is a submodule of $\Omega(b)$ which is generated by the rows of the matrix $\mathbf{K}_{\boldsymbol{b}}$ and is often called the module of Koszul relations of $\boldsymbol{b}$.

It is easy to see that $\Psi_{\boldsymbol{a}}(K(\boldsymbol{b}))=\mathfrak{a}$, since, for example, $\boldsymbol{a} \times\left(0, b_{3},-b_{2}\right)=a_{1} \boldsymbol{b}$ and $\Psi_{a}\left(\left(0, b_{3},-b_{2}\right)\right)=a_{1}$.

Lemma (2.8). We have the equality $\Psi_{a}^{-1}(\mathfrak{a})=K(\boldsymbol{b})+R a$.

Proof. Since $\Psi_{a}(a)=0$ by definition, it follows from the previous remark that $\Psi_{a}^{-1}(\mathfrak{a}) \supseteq K(\boldsymbol{b})+R a$. To show the opposite inclusion, let $x \in \Psi_{a}^{-1}(\mathfrak{a})$. Since we can find $y \in F$ with $\Psi_{a}(x)=a \cdot y$, we have by definition that $a \times x=(a \bullet y) b$ $=\boldsymbol{a} \times(\boldsymbol{b} \times \boldsymbol{y})$. Thus $\boldsymbol{x}-\boldsymbol{b} \times \boldsymbol{y}$ lies in the kernel of the map $\boldsymbol{a} \times$, and hence in Ra. Therefore $x \in K(b)+R a$ as desired.

Next we remark on the image and the cokernel of the map $d_{\boldsymbol{a}}: H^{3}(\boldsymbol{b}) \rightarrow H^{2}(\boldsymbol{b})$ defined in $\S 1$. See also Remark (1.10).

Lemma (2.9). Let $\mathfrak{a}^{\prime}$ be the ideal $\delta_{a}^{* *}\left(M^{* *}\right)$ of $R$. Then we have an isomorphism $\operatorname{Coker}\left(d_{\boldsymbol{a}}\right) \cong \mathfrak{a}^{\prime} / \mathfrak{a}$.

Proof. First of all note that, by definition, $H^{2}(b)$ is equal to the module $\Omega(b) / K(b)$ and $\operatorname{Im}\left(d_{a}\right)=R a+K(b) / K(b)$. On the other hand, (2.6) states that the image of the map $\Psi_{a}: \Omega(b) \rightarrow R$ is just $\mathfrak{a}^{\prime}$. Therefore the lemma follows immediately from (2.8).

Now changing the roles of $a$ and $b$, we may consider the module $\Omega(a)$ 
and the map $\Psi_{b}: \Omega(a) \rightarrow R$.

Lemma (2.10). Let us denote the inage of $\Psi_{b}$ by $\mathfrak{b}^{\prime}$. Then we have $\operatorname{Ker}\left(d_{\boldsymbol{a}}\right) \cong \mathfrak{b}^{\prime} / \mathfrak{b} \subseteq H^{3}(\boldsymbol{b}) \cong R / \mathfrak{b}$, and therefore $\operatorname{Im}\left(d_{\boldsymbol{a}}\right) \cong R / \mathfrak{b}^{\prime}$.

Proof. It is obvious that $H^{3}(\boldsymbol{b}) \cong R / \mathfrak{b}$ and $H^{2}(\boldsymbol{b})=\Omega(\boldsymbol{b}) / K(\boldsymbol{b})$. Furthermore, under this isomorphism, $d_{\boldsymbol{a}}: H^{3}(\boldsymbol{b}) \rightarrow H^{2}(\boldsymbol{b})$ is the map sending the class of $\alpha \in R$ to the class of $\alpha \boldsymbol{a}$ in $\Omega(\boldsymbol{b}) / K(\boldsymbol{b})$. Thus it is enough to show that $\alpha \boldsymbol{a} \in K(\boldsymbol{b})$ if and only if $\alpha \in \Psi_{b}(\Omega(a))$.

If $\alpha \boldsymbol{a} \in K(\boldsymbol{b})$, then we have $\alpha \boldsymbol{a}=\boldsymbol{b} \times \boldsymbol{x}$ for some $\boldsymbol{x} \in F$. Then, by (2.3), $(\boldsymbol{a} \cdot \boldsymbol{x}) \boldsymbol{b}=\boldsymbol{a} \times(\boldsymbol{b} \times \boldsymbol{x})=\boldsymbol{a} \times \alpha \boldsymbol{a}=0$, hence $\boldsymbol{a} \cdot \boldsymbol{x}=0$. This implies that $\boldsymbol{x} \in \Omega(\boldsymbol{a})$ and that $\Psi_{b}(x)=\alpha$. Hence $\alpha \in \Psi_{b}(\Omega(a))$. The converse is proved similarly.

Together with Theorem (1.11), these results are illustrated as the following diagram in which all the slanting sequence are exact:

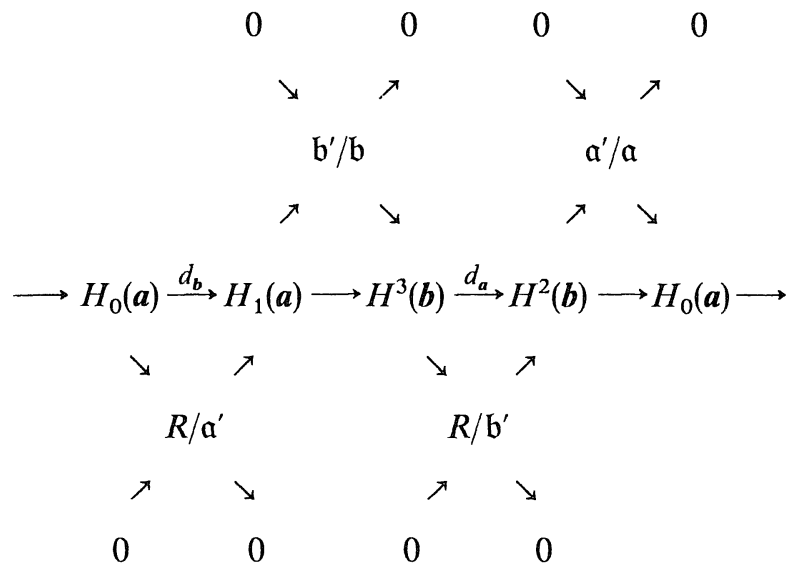

\section{§3. Homogeneity}

In the rest of the paper, $(R, \mathrm{~m}, k)$ always denotes a complete local hypersurface ring which is a non-regular, normal domain and of dimension two. Furthermore we assume that $k$ is an algebraically closed field of characteristic 0 .

In other words, $R$ is a residue ring of the formal power series ring $P=k[[X, Y, Z]]$ by an equation $f ; R=P / f P$. Since $R$ is a non-regular ring, denoting the maximal ideal of $P$ by $\mathfrak{m}_{P}$, we must have $f \in \mathfrak{m}_{P}^{2}$. Also we remark that the Jacobian ideal $\left(f_{X}, f_{Y}, f_{Z}\right) R$ is an m-primary ideal, since $R$ is normal 
hence it has only an isolated singularity.

Following the notation in $\S 2$, we set $F=R^{3}, \nabla f=\left(f_{X}, f_{Y}, f_{Z}\right) \in F$ and $J=\left(f_{X}, f_{Y}, f_{Z}\right) R$. We shall apply the results in the previous sections by putting $B=\nabla \mathfrak{f}$ and $\mathfrak{b}=J$.

Recall that the universally finite module of differentials $D=D_{k}(R)$ is given by the exact sequence:

$$
0 \rightarrow R \stackrel{\nabla f}{\rightarrow} F \rightarrow D \rightarrow 0
$$

Taking $R$-dual, we immediately obtain $\operatorname{Der}_{k}(R, R) \cong D^{*} \cong \Omega(\nabla f) \subseteq F$. (See Remark (2.5) for the definition of $\Omega$.)

Remark (3.1).

(3.1.1) As an ideal of $R, J$ cannot be generated by less than three elements.

(3.1.2) Whenever $R$ is not quasi-homogeneous, the ideal $\left(f, f_{X}, f_{Y}, f_{Z}\right) P$ of $P$ cannot be generated by less than four elements.

\section{Proof.}

(3.1.1) Assume the contrary, and let $f_{Z}=\alpha f_{X}+\beta f_{Y}(\alpha, \beta \in R)$ for example. Then the following complex:

$$
0 \longrightarrow R^{2} \stackrel{\left(\begin{array}{ccc}
-f_{X} & f_{Y} & 0 \\
-\alpha & -\beta & 1
\end{array}\right)}{\longrightarrow} R^{3} \stackrel{\left(\begin{array}{c}
f_{X} \\
f_{Y} \\
f_{Z}
\end{array}\right)}{\longrightarrow} R
$$

is exact. Indeed, since $\mathbb{I}_{2}\left(\begin{array}{ccc}-f_{X} & f_{Y} & 0 \\ -\alpha & -\beta & 1\end{array}\right)=\left(f_{X}, f_{Y}\right)=J$ is m-primary, a result of Buchsbaum-Eisenbud [3] shows that the sequence is exact. Thus it is observed that $D^{*} \cong \Omega(\nabla f)$ is isomorphic to $R^{2}$. Hence the Zariski-Lipman conjecture which is established for hypersurfaces ([9]) implies that $R$ would be regular. This is against the assumption.

(3.1.2) Let $J^{\prime}=\left(f, f_{X}, f_{Y}, f_{Z}\right) P$. Then, since $J=J^{\prime} / f P$, (3.1.1) shows $\operatorname{dim}_{k}\left(J^{\prime} / f P\right.$ $\left.+m_{P} J^{\prime}\right)=3$. Hence we have only to show that $f \notin m_{P} J^{\prime}$, for then $\operatorname{dim}_{k}\left(J^{\prime} / \mathrm{m} J^{\prime}\right)=4$. But this is almost immediate, since if $f \in m_{P} J^{\prime}$ then $f \in\left(f_{X}, f_{Y}, f_{Z}\right) P$ and a theorem 
of Saito [8] shows that $R$ would be quasi-homogeneous.

Lemma (3.2). The following two conditions are equivalent for $R$.

(3.2.1) There is an $R$-homomorphism $\varepsilon: D^{* *} \rightarrow R$ whose image is just $\mathrm{m}$.

(3.2.2) There is an element $\boldsymbol{a}=\left(a_{1}, a_{2}, a_{3}\right) \in \Omega(\nabla \boldsymbol{f})$ such that $\mathfrak{a}=\left(a_{1}, a_{2}, a_{3}\right) R$ is $\mathrm{m}$-primary and that $\Psi_{a}(\Omega(\nabla f))=\mathrm{m}$.

Moreover if it is the case, we have $\mathfrak{a}=\varepsilon(D)$.

Proof. (3.2.1) $\Rightarrow(3.2 .2)$ : We consider the restriction of $\varepsilon$ to $D$ through the natural map $D \subseteq D^{* *}$, which we denote by $\gamma$. Note that $\gamma^{* *}=\varepsilon$ hence the image of $\gamma$ is an m-primary ideal of $R$.

As $D$ is the residue module of $F$ by a submodule $R \nabla f$, we can find an element $\boldsymbol{a}=\left(a_{1}, a_{2}, a_{3}\right) \in \Omega(\nabla \boldsymbol{f})$ that makes the following digram commutative:

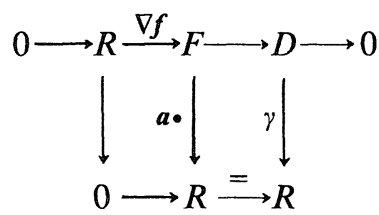

In particular we see that $\boldsymbol{a} \cdot \nabla \boldsymbol{f}=0$ hence $\boldsymbol{a} \in \boldsymbol{\Omega}(\boldsymbol{\nabla} \boldsymbol{f})$, and moreover $\left(a_{1}, a_{2}, a_{3}\right) R$ $=\operatorname{Im}(\gamma)$ is an m-primary ideal. (So the conditions (2.1.1) and (2.1.2) are satisfied.) It is easily observed that $\gamma$ is equal to $\delta_{a}$ in the notation of $\S 1$, hence that $\varepsilon=\delta_{a}^{* *}$. Thus Lemma (2.6) yields (3.2.2).

The converse will also follow, but more easily, from (2.6).

Note that the equality $\Psi_{a}(\Omega(\nabla f))=R$ never occurs for any choice of $a \in \Omega(\nabla f) . \quad$ In fact, if it does, then from the same argument as in showing (0.2) we will have $D^{* *} \cong \Omega(\nabla f) \cong R^{2}$, and it thus follows from the Zariski-Lipman conjecture that $R$ would be a regular local ring, contradicting our assumption.

Lemma (3.3). Let $a=\left(a_{1}, a_{2}, a_{3}\right)$ be an element in $\Omega(\nabla \boldsymbol{f})$ and suppose that $\mathfrak{a}=\left(a_{1}, a_{2}, a_{3}\right) R$ is an m-primary ideal. Then the following two conditions are equivalent.

$$
\Psi_{a}(\Omega(\nabla f))=\mathfrak{m} .
$$

(3.3.2) $\nabla f$ represents a nontrivial socle element in $H_{1}(a)=\Omega(a) / K(a)$. 
Proof. Before proceeding to the proof, we note that we may apply the results in the previous sections to the situation $\boldsymbol{a}$ and $\boldsymbol{b}=\nabla \boldsymbol{f}$, hence $M=D$. (3.3.1) $\Rightarrow$ (3.3.2): As to the maps $d_{\boldsymbol{a}}: H^{3}(\nabla \boldsymbol{f}) \rightarrow H^{2}(\nabla \boldsymbol{f})$ and $d_{\nabla f}: H_{0}(\boldsymbol{a}) \rightarrow H_{1}(\boldsymbol{a})$, we have shown in (1.11) and (2.9) that $\operatorname{Ker}\left(d_{\nabla \boldsymbol{f}}\right) \cong \operatorname{Coker}\left(d_{\boldsymbol{a}}\right) \cong \mathfrak{m} / \mathfrak{a}$. Thus, noting that the map $d_{\nabla \boldsymbol{f}}$ sends the class of 1 in $R / \mathfrak{a}=H_{0}(\boldsymbol{a})$ to the class of $\nabla \boldsymbol{f}$ in $\Omega(\boldsymbol{a}) / K(\boldsymbol{a})=H_{1}(\boldsymbol{a})$, we see that $\nabla \boldsymbol{f}$ generates in $H_{1}(\boldsymbol{a})$ the module $\operatorname{Im}\left(d_{\nabla \boldsymbol{f}}\right) \cong R / \mathrm{m}$. This shows (3.3.2).

(3.3.2) $\Rightarrow$ (3.3.1): By the condition (3.3.2) we see that the image of the map $d_{\nabla \boldsymbol{f}}: H_{0}(\boldsymbol{a}) \rightarrow H_{1}(\boldsymbol{a})$ is isomorphic to $R / \mathrm{m}$. Therefore it follows from (2.11) that $R / \mathfrak{a}^{\prime} \cong \operatorname{Im}\left(d_{\nabla f}\right) \cong R / \mathfrak{m}$, where $\mathfrak{a}^{\prime}=\operatorname{Im}\left(\delta_{a}^{* *}\right)$ as in (2.9). Thus, comparing the length of the both sides we have $\operatorname{Im}\left(\delta_{a}^{* *}\right)=\mathrm{m}$.

We are summing up the above two lemmas to get the following:

Theorem (3.4). The following three conditions are equivalent for $R$.

(3.4.1) There is a surjective R-homomorphism $D^{* *} \rightarrow \mathrm{m}$.

(3.4.2) There exists an element $\boldsymbol{a}=\left(a_{1}, a_{2}, a_{3}\right)$ of $F$ which satisfies:

(3.4.2a) $a \in \Omega(\nabla f)$ and $\mathfrak{a}=\sum_{i=1}^{3} a_{i} R$ is an m-primary ideal.

(3.4.2b) $\quad \Psi_{a}(\Omega(\nabla f))=m$

(3.4.3) There is an element $\boldsymbol{a}=\left(a_{1}, a_{2}, a_{3}\right)$ of $F$ with the condition (3.4.2a) and it satisfies:

(3.4.3b) $\nabla f$ represents a nontrivial socle of $H_{1}(a)=\Omega(a) / K(a)$.

Definition (3.5). For the brevity, we refer to each condition in Theorem (3.4) as the condition (A). Moreover if $\boldsymbol{a}$ is taken as (3.4.2) and (3.4.3) are fullfiled, then we say that the condition (A) is satisfied with $a$.

Recall from $\S 0$ that the conjecture $(0.3)$ asks if $R$ is quasi-homogeneous under the condition (A).

Proposition (3.6). Suppose $R$ satisfies the condition $(A)$ with a. Then for any $\boldsymbol{x}=(\xi, \eta, \zeta) \in F$ where $\{\xi, \eta, \zeta\}$ is a system of generators for $\mathfrak{m}$, there exist $\mathbf{g} \in F$ and $\mathbf{H} \in \operatorname{End}_{R}(F)$ that make the following diagram commutative: 


$$
0 \longrightarrow R \stackrel{a}{\longrightarrow} F \stackrel{a \times}{\longrightarrow} F \stackrel{a \bullet}{\longrightarrow} R \longrightarrow 0
$$

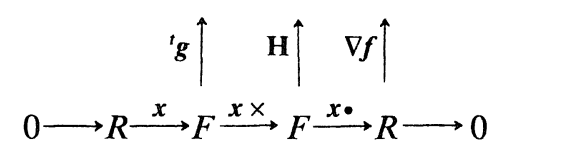

Proof. By the condition (3.4.3b), all of $\xi \nabla f, \eta \nabla f$ and $\zeta \nabla f$ belong to the image of $a \times: F \rightarrow F$. Therefore we have $\operatorname{Im}\left({ }^{t} x \nabla f\right) \subseteq \operatorname{Im}(a \times)$. Then the proposition is almost immediate if we notice that part $0 \rightarrow R \rightarrow F \rightarrow F$ of the first row is exact and that the second row is a complex of free modules.

Notation and Remark (3.7). For a given $x \in F$ as in (3.6), we denote the rows of the matrix $\mathbf{H}$ by $\boldsymbol{c}_{\xi}, \boldsymbol{c}_{\eta}$, and $\boldsymbol{c}_{\zeta}$ :

$$
\mathbf{H}=\left(\begin{array}{c}
c_{\xi} \\
c_{\eta} \\
c_{\zeta}
\end{array}\right)
$$

Also we denote $\boldsymbol{g}=\left(g_{1}, g_{2}, g_{3}\right)$.

The commutativity of the diagram (3.6.1) is equivalent to the following equations:

$$
\begin{aligned}
& \text { (3.7.1) } \zeta \nabla f=a \times c_{\xi}, \quad \eta \nabla f=a \times c_{\eta}, \quad \zeta \nabla f=a \times c_{\zeta}, \\
& \text { (3.7.2) } \quad \zeta c_{\eta}-\eta c_{\zeta}=g_{1} a, \quad-\zeta c_{\xi}+\xi c_{\zeta}=g_{2} a, \quad \eta c_{\xi}-\xi c_{\eta}=g_{3} a .
\end{aligned}
$$

Moreover they induce the following equations:

$$
c_{\xi} \times c_{\eta}=g_{3} \nabla f, \quad c_{\eta} \times c_{\zeta}=g_{1} \nabla f, \quad c_{\zeta} \times c_{\xi}=g_{2} \nabla f
$$

These data have good information on $\Omega(\nabla f)$ as we show in the next lemma.

Lemma (3.8). Suppose the condition $(A)$ is satisfied with a. Then under the notation as in (3.7), $\Omega(\nabla f)$ is generated by the four elements $a, c_{\xi}$, $c_{\eta}$ and $c_{\zeta}$.

Proof. It follows from (3.7.1) that

$$
\xi \nabla f \cdot c_{\xi}=\left(a \times c_{\xi}\right) \cdot c_{\xi}=0,
$$

thus $\nabla \boldsymbol{f} \cdot c_{\xi}=0$, for $\xi$ is a non zero divisor on $R$. Consequently we have $c_{\xi} \in \Omega(\nabla f)$. Likewise we see that $\left\{a, c_{\xi}, c_{\eta}, c_{\zeta}\right\} \subseteq \Omega(\nabla f)$. 
We want to show that these four elements actually generate the module $\Omega(\nabla f)$. For this let $y \in \Omega(\nabla f)$. Then by the definition of $\Psi_{a}: \Omega(\nabla f) \rightarrow R$, we have $a \times y=\Psi_{a}(y) \nabla f$. Here we note from (3.4.2b) that $\Psi_{a}(y) \in \mathrm{m}$. Thus we can find $\alpha_{1}, \alpha_{2}, \alpha_{3} \in R$ so that $\Psi_{a}(y)=\alpha_{1} \xi+\alpha_{2} \eta+\alpha_{3} \zeta$. Then by the equations (3.7.1) we see that $a \times y=a \times\left(\alpha_{1} c_{\xi}+\alpha_{2} c_{\eta}+\alpha_{3} c_{\zeta}\right)$, hence $y-\left(\alpha_{1} c_{\xi}+\alpha_{2} c_{\eta}+\alpha_{3} c_{\zeta}\right)$ $\in \operatorname{Ker}(a \times)=R a$. This shows the desired result.

More precisely than this lemma, we shall show in (3.11) that the four elements are minimally generating $\Omega(\nabla f)$. To do this, we need some auxiliary results.

Note that it is known and is easy to see that there is an isomorphism $H_{1}(x) \cong\left(m_{P}: f\right) / m_{P}$ and the last module is isomorphic to $k$. This is indeed characterizing hypersurfaces. See for instance [4, Proposition 3.3.4].

Lemma (3.9). Suppose the condition $(A)$ is satisfied with a. Then under the notation in (3.7), $g$ represents a nontrivial element, hence a generator, of $H_{1}(\boldsymbol{x})$. Thus $f$ is described as $f=g_{1} \xi+g_{2} \eta+g_{3} \zeta$ in $P$ up to units.

Proof. Suppose that $g$ represents a trivial element in $H_{1}(x)$. Then we have $g \in \operatorname{Im}(x \times)$, and thus by chasing diagram, we can construct a chain homotopy of the chain map (that is the transpose of (3.6.1)):

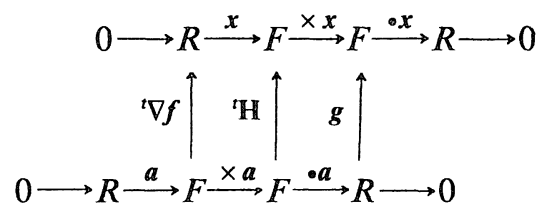

In particular there is $y \in F$ such that ${ }^{t} \nabla f: F \rightarrow R$ is a composition of $\times a: F \rightarrow F$ with ${ }^{t} y: F \rightarrow R$. But this means that $\nabla f$ is in the image of $a \times: F \rightarrow F$ and it contradicts (3.4.3b). Therefore $g$ must be non-trivial as an element in $H_{1}(\boldsymbol{x})$.

It is known that the last statement follows from the first. See [4, Prop. 1.5.4].

Remark (3.10). Under the situation as in (3.6), if we are given another expression $f=\boldsymbol{g}^{\prime} \cdot \boldsymbol{x}=g_{1}^{\prime} \xi+g_{2}^{\prime} \eta+g_{3}^{\prime} \zeta$ in $P$, we can take $\boldsymbol{g}^{\prime}$ in place of $\boldsymbol{g}$ with taking another $\mathbf{H}^{\prime} \in \operatorname{End}_{R}(F)$ instead of $\mathbf{H}$.

Proof. The assumption $\boldsymbol{g}^{\prime} \cdot \boldsymbol{x}=\boldsymbol{g} \cdot \boldsymbol{x}$ in $P$ is equivalent to saying that 
$\boldsymbol{g}-\boldsymbol{g}^{\prime}=\boldsymbol{x} \times \boldsymbol{y}$ in $P^{3}$ for some $\boldsymbol{y} \in P^{3}$. (Note that $\{\xi, \eta, \zeta\}$ is a regular sequence on $P$.) Considering the matrix $\mathbf{H}^{\prime}=\mathbf{H}+{ }^{t} \boldsymbol{y} \boldsymbol{a}$ on $R$, we have

$$
{ }^{t} g^{\prime} \boldsymbol{a}={ }^{t}(\boldsymbol{g}-\boldsymbol{x} \times \boldsymbol{y}) \boldsymbol{a}=\mathbf{K}_{\boldsymbol{x}} \mathbf{H}+\mathbf{K}_{\boldsymbol{x}}{ }^{t} \boldsymbol{y} \boldsymbol{a}=\mathbf{K}_{\boldsymbol{x}} \mathbf{H}^{\prime},
$$

and $\mathbf{H}^{\prime} \mathbf{K}_{\boldsymbol{a}}=\mathbf{H K}_{\boldsymbol{a}}$. Hence we may replace $\boldsymbol{g}$ with $\boldsymbol{g}^{\prime}$.

As for the number of generators of $\Omega(\nabla f)$ which is four at most by Lemma (3.8), we show it is precisely four.

Proposition (3.11). Suppose the condition $(A)$ is satisfied with a. Then $\left\{\boldsymbol{a}, \boldsymbol{c}_{\xi}, \boldsymbol{c}_{\eta}, \boldsymbol{c}_{\zeta}\right\}$ minimally generates $\Omega(\nabla \boldsymbol{f})$.

Proof. First we show that $a$ is part of minimal generating system of $\Omega(\nabla f)$.

Suppose the contrary, and assume that

$$
a=\beta_{1} c_{\xi}+\beta_{2} c_{\eta}+\beta_{3} c_{\zeta}
$$

for some $\beta_{1}, \beta_{2}, \beta_{3} \in R$. Then applying $\times c_{\xi}$ on both sides, we get from (3.7.1) and (3.7.3) that $\xi=-g_{3} \beta_{2}+g_{2} \beta_{3}$. Since $f \in m_{P}^{2}$ and since $f=g_{1} \xi+g_{2} \eta+g_{3} \zeta$ up to units, for one of the choices we may take $g$ as $g \in m F$. Thus by Remark (3.10), replacing $g$ if necessary, we may assume that $g_{i} \in \mathfrak{m}(1 \leq i \leq 3)$. Therefore one of $\beta_{2}$ and $\beta_{3}$ has to be a unit. Actually, if not then $\xi=-g_{3} \beta_{2}+g_{2} \beta_{3} \in \mathfrak{m}^{2}$, contradicting that $\xi, \eta, \zeta$ minimally generate $\mathrm{m}$. Without loss of generality we assume $\beta_{3}$ is a unit. Then operating $a \times$ on the both sides of (3.11.1), we get $0=\beta_{1} \xi+\beta_{2} \eta+\beta_{3} \zeta$, and thus $\zeta \in(\xi, \eta) R$, a contradiction.

Now we prove the proposition. For this, we consider the map

$$
\Psi_{a} \otimes_{R} k: \Omega(\nabla \boldsymbol{f}) / \mathrm{m} \Omega(\nabla \boldsymbol{f}) \rightarrow \mathrm{m} / \mathrm{m}^{2},
$$

which is surjective by the condition (A). Since $\Psi_{a}(a)=0$ by definition and since $\operatorname{dim}_{k}\left(m / m^{2}\right)=3$, we see from the above claim that $\operatorname{dim}_{k}(\Omega(\nabla f) / m \Omega(\nabla f)) \geq 4$. This shows that $\Omega(\nabla f)$ never be generated by less than four elements. Thus the proposition follows from (3.8).

Making thorough use of the properties of differential modules, we may give a sufficient condition for $R$ to be quasi-homogeneous.

Theorem (3.12). Suppose the condition $(A)$ is satisfied with a. And suppose that a represents an element of $\mathrm{m} H_{1}(\nabla f)$. Then $R$ is quasi-homogeneous. 
Proof. Recall that $H_{1}(\nabla f)=\Omega(\nabla f) / K(\nabla f)$. Therefore the assumption says that $a \in \mathfrak{m} \Omega(\nabla \boldsymbol{f})+K(\nabla \boldsymbol{f})$. Thus we may write $\boldsymbol{a}=\boldsymbol{a}^{\prime}+\nabla \boldsymbol{f} \times \boldsymbol{y}$ for some $\boldsymbol{a}^{\prime} \in \mathrm{m} \Omega(\nabla \boldsymbol{f})$ and $y \in F$. Here we also may assume that $y \in k^{3}$, because $F=m F+k^{3}$ and $\nabla f \times m F \subseteq m \Omega(\nabla f)$.

Now we show that one can take $\boldsymbol{a}^{\prime}=0$. In fact, it is easily observed that $\Psi_{a}=\Psi_{a^{\prime}}+\Psi_{\nabla f \times y}$, and that $\Psi_{a^{\prime}}(\Omega(\nabla f)) \in m^{2}$ (by the remark after Lemma (3.2)). So the condition (3.4.2b) implies

$$
m=\Psi_{a}(\Omega(\nabla f)) \subseteq \Psi_{\nabla f \times y}(\Omega(\nabla f))+m^{2} .
$$

Consequently, $\Psi_{\nabla \boldsymbol{f} \times \boldsymbol{y}}(\Omega(\nabla f))=\mathfrak{m}$ by Nakayama's lemma. Thus the condition (3.4.2b) is satisfied with $\boldsymbol{a}=\nabla \boldsymbol{f} \times \boldsymbol{y}$, and in this case $\boldsymbol{a}$ represents a trivial element of $H_{1}(\nabla f)$.

To show that the three components of $\nabla \boldsymbol{f} \times \boldsymbol{y}$ generate an m-primary ideal, we denote $y=\left(\lambda_{1}, \lambda_{2}, \lambda_{3}\right)$ with $\lambda_{i} \in k$. We remark that $y \neq 0$, since otherwise $\Psi_{\nabla \boldsymbol{f} \times \boldsymbol{y}}(\Omega(\nabla \boldsymbol{f}))=0$. Now take a $3 \times 3$ matrix

$$
\Lambda=\left(\begin{array}{lll}
\lambda_{1} & \lambda_{2} & \lambda_{3} \\
\mu_{1} & \mu_{2} & \mu_{3} \\
v_{1} & v_{2} & v_{3}
\end{array}\right)
$$

whose entries are in $k$ and such that $\Lambda$ is symmetric and invertible.

Change a system of generators for $m=(X, Y, Z) R=(U, V, W) R$ so as $(U, V, W)=(X, Y, Z) \Lambda$ in $F$. And note that $\left(f_{X}, f_{Y}, f_{Z}\right)=\left(f_{U}, f_{V}, f_{W}\right)^{t} \Lambda$, and for elements $(x, y, z),(u, v, w) \in F$ with the relation $(u, v, w)=(x, y, z) \Lambda$, we have $x f_{X}+y f_{Y}+z f_{Z}=u f_{U}+v f_{V}+w f_{W}$. Therefore,

$$
\begin{aligned}
\Psi_{\nabla \boldsymbol{f} \times \boldsymbol{y}}(\Omega(\nabla \boldsymbol{f})) & =\left\{x \lambda_{1}+y \lambda_{2}+z \lambda_{3} \mid x f_{X}+y f_{Y}+z f_{Z}=0\right\} \\
& =\left\{u \mid u f_{U}+v f_{V}+w f_{W}=0\right\} \\
& =\left(f_{V}, f_{W}\right): f_{U}
\end{aligned}
$$

After all, changing variables from $X, Y, Z$ to $U, V, W$, we may take $\left(0, f_{Z},-f_{Y}\right)=\nabla f \times(1,0,0)$ as $\nabla \boldsymbol{f} \times \boldsymbol{y}$. And by the above equality, we have $\mathrm{m}=\left(f_{\mathrm{Y}}, f_{Z}\right): f_{X}$. From this we can easily see that $\left\{f_{Y}, f_{Z}\right\}$ generate an m-primary ideal of $R$. Indeed, if $\left(f_{Y}, f_{Z}\right) R$ were contained in a prime ideal $\mathfrak{p}$ of height one, then that $f_{X} \mathrm{~m} \subseteq\left(f_{Y}, f_{Z}\right) R \subseteq \mathfrak{p}$ would imply that $f_{X} \in \mathfrak{p}$, and this contradicts the fact that $\left\{f_{X}, f_{Y}, f_{Z}\right\}$ generate an m-primary ideal of $R$.

Therefore we conclude that the condition (A) is satisfied with $\nabla f \times y$, thus 
we may assume that $a=\nabla f \times y$ with $y=(1,0,0) \in k^{3}$.

For simplification, we introduce the notation $\boldsymbol{k}_{X}, \boldsymbol{k}_{Y}, \boldsymbol{k}_{Z}$ for three generators of $K(\nabla \boldsymbol{f})$, that is, $\boldsymbol{k}_{X}=\nabla \boldsymbol{f} \times(1,0,0)=\left(0, f_{Z},-f_{Y}\right), \boldsymbol{k}_{Y}=\nabla \boldsymbol{f} \times(0,1,0)=\left(-f_{Z}, 0, f_{X}\right)$ and $\boldsymbol{k}_{Z}=\nabla \boldsymbol{f} \times(0,0,1)=\left(f_{Y},-f_{X}, 0\right)$ as elements in $F$.

Now proceed with our argument in setting

$$
\boldsymbol{a}=\boldsymbol{k}_{X}, \quad \text { hence } \quad \boldsymbol{a}=\left(f_{Y}, f_{Z}\right) R,
$$

and in supposing that $R$ is not quasi-homogeneous. We aim at deriving a contradiction from this situation.

First we write part of the diagram in (1.9) as follows:

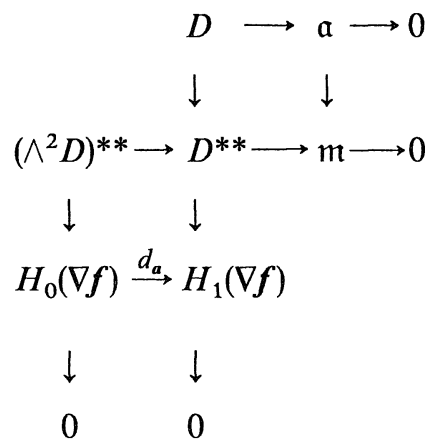

where the middle row is exact. (To get this daigram from (1.9) note that $\operatorname{Im}\left(\delta_{a}\right)=\mathfrak{a}$ and $\operatorname{Im}\left(\delta_{a}^{* *}\right)=m$ from (3.4) and identify $H_{i}(\nabla f)$ with $H^{3-i}(\nabla f)$ for $0 \leq i \leq 3$.) It follows from this that there is an exact sequence:

$$
H_{0}(\nabla f) \stackrel{d_{a}}{\rightarrow} H_{1}(\nabla f) \rightarrow \mathrm{m} / \mathfrak{a} \rightarrow 0 .
$$

Note however that $H_{0}(\nabla f) \cong R / J$ and that the map $d_{a}$ sends the generator of $H_{0}(\nabla f)$ to the class of $a$ in $H_{1}(\nabla f)$, thus it follows that $d_{a}=0$, since we have assumed $a=k_{x}$ that is trivial as an elements of $H_{1}(\nabla f)$. As a consequence we have

$$
H_{1}(\nabla f) \cong m / \mathfrak{a} .
$$

On the other hand, since $R$ is assumed to be non-quasi-homogeneous, it is known from (3.1.2) that the ideal $\left(f, f_{X}, f_{Y}, f_{Z}\right) P$ of $P$ is minimally generated by four elements. Thus the Artinian ring $R / J=P /\left(f, f_{X}, f_{Y}, f_{Z}\right) P$ is an almost complete intersection. It then follows that the canonical module $K_{R / J}$ of $R / J$ 
is obtained as the Koszul homology $H_{1}(\nabla f)$.

Combining this with (3.12.2), we consequently have

$$
K_{R / J} \cong \mathfrak{m} / \mathfrak{a} .
$$

Secondly we claim that

$$
\operatorname{Soc}(R / \mathfrak{a})=J / \mathfrak{a} \quad \text { and } \quad l(J / \mathfrak{a})=1,
$$

where $S o c$ denotes the socle of a module and $l$ denotes the length. To prove this, let us notice that the canonical module is a faithful module, hence using (3.12.3) we see $J=\operatorname{ann}_{R}\left(K_{R / J}\right)=a n n_{R}(\mathfrak{m} / \mathfrak{a})=\left(\mathfrak{a}:{ }_{R} \mathfrak{m}\right)$. The first equality of (3.12.4) follows from this. On the other hand, it is known that $R / J$ and $K_{R / J}$ have the same length, and thus $l(R / J)=l(\mathfrak{m} / \mathfrak{a})$. Since there are inclusions $\mathfrak{a} \subseteq J \subseteq \mathfrak{m} \subset R$, it follows that $l(J / \mathfrak{a})=1$.

Note that (3.12.4) means exactly that $R / \mathfrak{a}$ is a Gorenstein ring whose one-dimensional socle is $J / \mathfrak{a}$. Since we have assumed that $\mathfrak{a}=\left(f_{Y}, f_{Z}\right) R$, we must have

$$
J / \mathfrak{a} \text { is generated by the class of } f_{X} .
$$

Therefore $f_{X}$ is to generate the socle of $R / \mathfrak{a}=R /\left(f_{Y}, f_{Z}\right) R=P /\left(f, f_{Y}, f_{Z}\right) P$. On the other hand, since $\left\{f, f_{Y}, f_{Z}\right\}$ is a regular sequence in $P$, it is known by $[10$, Korollar 4.7] that the socle of $P /\left(f, f_{Y}, f_{Z}\right) P$ is gotten by their Jacobian:

$$
\left|\begin{array}{lll}
f_{X} & f_{Y} & f_{Z} \\
f_{Y X} & f_{Y Y} & f_{Y Z} \\
f_{Z X} & f_{Z Y} & f_{Z Z}
\end{array}\right|=f_{X}\left|\begin{array}{ll}
f_{Y Y} & f_{Y Z} \\
f_{Z Y} & f_{Z Z}
\end{array}\right| \quad \bmod \quad \mathfrak{a} .
$$

Since $f_{X}$ itself is the socle, the determinant $\left|\begin{array}{ll}f_{Y Y} & f_{Y Z} \\ f_{Z Y} & f_{Z Z}\end{array}\right|$ must be a unit in $P$. But this determinant is the Jacobian of the system of parameters $\left\{X, f_{Y}, f_{Z}\right\}$ relative to the variables $\{X, Y, Z\}$, thus it is concluded that $m_{P}=\left(X, f_{Y}, f_{Z}\right) P$. As a result, $P /\left(f_{Y}, f_{Z}\right) P \cong k[[X]]$ is a principal ideal domain. Therefore any ideal of $P$ containing $\left(f_{Y}, f_{Z}\right) P$ is generated by at most three elements, in particular $\left(f, f_{X}, f_{Y}, f_{Z}\right) P$ is. This contradicts the Remark (3.1.2), hence the proof is completed.

For a finitely generated $R$-module $M$, we denote by $\mu(M)$ the minimal 
number of generators for $M$. The Proposition (3.8) shows that $\mu\left(H_{1}(\nabla f)\right)$ is at most four.

Proposition (3.13). Suppose the condition $(A)$ is satisfied with a. Then the following equation holds:

$$
\mu\left(H_{1}(\nabla \boldsymbol{f})\right)=\mu(\mathrm{m} / \mathfrak{a})+s(\boldsymbol{a}),
$$

where the integer $s(\boldsymbol{a})$ is defined as:

$$
s(a)= \begin{cases}0 & \left(\text { if a represents an element in } m H_{1}(\nabla f)\right) \\ 1 & \text { (otherwise) }\end{cases}
$$

Proof. Let $I$ be the ideal $\Psi_{\nabla f}(\Omega(a)) \subseteq R$. Applying (2.11) to $b=\nabla f$, we obtain the exact sequence as below:

$$
0 \rightarrow R / I \rightarrow H_{1}(\nabla f) \rightarrow \mathfrak{m} / \mathfrak{a} \rightarrow 0 .
$$

(Note that $\mathfrak{b}^{\prime}=I, \mathfrak{a}^{\prime}=m$ and that there is a natural isomorphism $H^{2}(\nabla f) \cong H_{1}(\nabla f)$.)

Notice that the map $R / I \rightarrow H_{1}(\nabla f)$ sends the class of 1 to the class of $a$ in $H_{1}(\nabla f)$. Therefore if the class of $a$ lies in $m H_{1}(\nabla f)$, then (3.13.1) shows that $\mu\left(H_{1}(\nabla f)\right)=\mu(\mathfrak{m} / \mathfrak{a})$, and otherwise $\mu\left(H_{1}(\nabla f)\right)=\mu(\mathfrak{m} / \mathfrak{a})+1$.

As a corollary of this, the following is of particular importance:

Corollary (3.14). Suppose the condition $(A)$ is satisfied. Then the following two conditions are equivalent:

(3.14.1) $R$ is quasi-homogeneous.

(3.14.2) $\mu\left(H_{1}(\nabla f)\right)=1$.

Proof. First we note that $R$ is quasi-homogeneous if and only if $m=a$ for some choice of $\boldsymbol{a}$ with which the condition (A) is satisfied.

Actually, if $R$ is quasi-homogeneous, then we have an Euler derivation $\Delta$ on $R$. Considering $\boldsymbol{a}=(\Delta(X), \Delta(Y), \Delta(Z)) \in F$, we see that $\boldsymbol{a} \in \Omega(\nabla \boldsymbol{f})$, furthermore by the property of Euler derivation, $\mathfrak{a}(=(\Delta(X), \Delta(Y), \Delta(Z)) R)=\mathrm{m}$. Conversely if $\mathrm{m}=\mathfrak{a}$ for some $a \in \Omega(\nabla f)$, then we see that $\delta_{\boldsymbol{a}}: D \rightarrow R$ has the image $m$. But this implies that $R$ is quasi-homogeneous by [11; Satz 5.5].

Now we prove the corollary. If $R$ is quasi-homogeneous, then by the 
above we have $\mu\left(H_{1}(\nabla f)\right)=s(a) \leq 1$ for some $a$. But we know that $H_{1}(\nabla f) \neq 0$, hence the inequality must be an equality. On the contrary, suppose that $R$ is not quasi-homogeneous. Then by Theorem (3.12) we see that $a$ would represent one of minimal generators of $H_{1}(a)$, hence $s(a)=1$. Since $l(m / a)>0$ as remarked above, it follows from (3.13) that $\mu\left(H_{1}(\nabla f)\right)>1$.

The following theorem will be useful in actual computation in the next section.

Theorem (3.15). Suppose the following two conditions for $R$ hold.

(3.15.1) $f \in \mathrm{m}_{P}^{3}$. (So the multiplicity of $R$ is not less than three.)

(3.15.2) $\mu\left(H_{1}(\nabla f)\right) \leq 3$.

Then the following condition also holds if the condition $(A)$ is satisfied.

$$
\left(J_{P}:_{P} f\right) f \nsubseteq m_{P}^{2} J_{P} \text { in } P . \quad\left(\text { Recall that } J_{P}=\left(f_{X}, f_{Y}, f_{Z}\right) P .\right)
$$

Proof. We assume that the condition (3.15.3) does not hold, i.e.

$$
\left(J_{P}:{ }_{P} f\right) f \subseteq \mathrm{m}_{P}^{2} J_{P} \text { in } P
$$

and we shall prove the theorem by showing the contradiction from the condition (A).

Before proving the theorem, we first claim that under the conditions (3.15.1) and $\neg(3.15 .3)$, we have $a_{i} \in \mathrm{m}^{2}(1 \leq i \leq 3)$ for any choice of $a=\left(a_{1}, a_{2}, a_{3}\right)$ with which the condition (A) holds.

To show this we note that there is an exact sequence:

$$
0 \longrightarrow H_{1}(\nabla f) \stackrel{\varphi}{\longrightarrow} P / J_{P} \stackrel{f}{\longrightarrow} P / J_{P}
$$

which is induced from the exact sequence of Koszul complexes: $0 \rightarrow K_{\bullet}(\nabla f ; P)$ $\stackrel{f}{\rightarrow} K_{\circ}(\nabla f ; P) \rightarrow K_{\circ}(\nabla f ; R) \rightarrow 0$. Thus $\varphi$ is defined as follows: For any $\boldsymbol{x}$ $=\left(x_{1}, x_{2}, x_{3}\right) \in \Omega(\nabla f)$, taking a lifting $\xi_{i}$ of each $x_{i}$ to $P$, we have $\xi_{1} f_{X}+\xi_{2} f_{Y}+\xi_{3} f_{Z}=t_{x} f$ for some $t_{x} \in P$, since $x \cdot \nabla f=0$ in $R$. Then $\varphi$ sends the class of $\boldsymbol{x}$ in $H_{1}(\nabla f)$ to $t_{x}\left(\bmod J_{P}\right)$ in $P / J_{P}$, which is easily seen to be independent of the choice of liftings. Considering the image of the class of $a$, we have $t_{a} \in\left(J_{P}:_{P} f\right)$ hence $t_{a} f \in \mathrm{m}_{P}^{2} J_{P}$ by $\neg(3.15 .3)$. Therefore we can find $\alpha_{i}^{\prime} \in \mathrm{m}_{P}^{2}$ 
$(1 \leq i \leq 3)$ so that $t_{a} f=\alpha_{1}^{\prime} f_{X}+\alpha_{2}^{\prime} f_{Y}+\alpha_{3}^{\prime} f_{Z}$. Let $a_{i}^{\prime}=\alpha_{i}^{\prime}(\bmod f P) \in R$ and let $\boldsymbol{a}^{\prime}=\left(a_{1}^{\prime}, a_{2}^{\prime}, a_{3}^{\prime}\right) \in F$. Note that $a_{i}^{\prime} \in \mathrm{mt}^{2}(1 \leq i \leq 3)$. Since the classes of $\boldsymbol{a}$ and $\boldsymbol{a}^{\prime}$ go to the same element $t_{\boldsymbol{a}}$ under the map $\varphi$, and since $\varphi$ is injective, we can find $\boldsymbol{y} \in F$ such that $\boldsymbol{a}=\boldsymbol{a}^{\prime}+\nabla \boldsymbol{f} \times \boldsymbol{y}$. Note from (3.15.1) that each component of $\nabla f$ is in $\mathrm{m}^{2}$. Therefore the above equality implies $a_{i} \in \mathrm{m}^{2}(1 \leq i \leq 3)$, establishing the above claim.

Now return to the proof of the theorem. First suppose that $R$ is quasi-homogeneous. Then, since $f \in J_{P}$, we have $J_{P}:_{P} f=P$, thus it follows from $\neg(3.15 .3)$ that $f \in \mathfrak{m}_{P}^{2} J_{P}$, which is a contradiction, because $f \in \mathfrak{m}_{P} J_{P}-\mathfrak{m}^{2} J_{P}$ by using the Euler derivation. Whence we may assume that $R$ is non-quasihomogeneous.

Recall from (3.11) that $\Omega(\nabla f)$ is generated exactly by four elements, while the assumption (3.15.2) obliges that $\Omega(\nabla f) / K(\nabla f)$ is generated by at most three elements. Hence there is an element $k \in K(\nabla \boldsymbol{f})$ such that $\boldsymbol{k} \notin \mathfrak{m} \Omega(\nabla \boldsymbol{f})$. Since $R$ is non-quasi-homogeneous, note from (3.12) that $a$ must be a minimal generator of $H_{1}(\nabla \boldsymbol{f})$, therefore we can take $\{\boldsymbol{a}, \boldsymbol{k}, \boldsymbol{x}, \boldsymbol{y}\}$ (for some $\boldsymbol{x}, \boldsymbol{y} \in F$ ) as a minimal system of generators of $\Omega(\nabla f)$. As $\Psi_{a}: \Omega(\nabla f) \rightarrow m$ is a surjective map from the condition (A), and as $\Psi_{a}(a)=0$, we must have that $\left\{\Psi_{a}(k), \Psi_{a}(x), \Psi_{a}(y)\right\}$ is a minimal system of generators of $m$, in particular $\Psi_{a}(k) \notin m^{2}$.

On the other hand, we may write $\boldsymbol{k}=c_{1} \boldsymbol{k}_{X}+c_{2} \boldsymbol{k}_{\mathrm{Y}}+c_{3} \boldsymbol{k}_{Z}$ for some $c_{i} \in R$ $(1 \leq i \leq 3)$. And noticing that $\boldsymbol{a} \times \boldsymbol{k}_{X}=a_{1} \nabla \boldsymbol{f}$ so on, we have $\Psi_{\boldsymbol{a}}(\boldsymbol{k})=c_{1} a_{1}+c_{2} a_{2}$ $+c_{3} a_{3}$, which is in $\mathrm{m}^{2}$ by the above claim. Thus we have a contradiction.

\section{§4. Examples}

As we mentioned in $\S 0$, we shall now look into some special cases where the conjecture $(0.3)$ is true. Following the notation in $\S 3$, we set $f \in P=k[[X, Y, Z]]$ and $R=P / f P$. Moreover we assume that $k$ is an algebraically closed field of characteristic 0 .

Example (4.1). (See also [6].) Let $f$ be of the form $g+X^{n}$ where $g \in k[[Y, Z]]$ and $n \geq 2$, and suppose that $R=P / f P$ is a normal domain. In this case, if $R$ satisfies the condition $(A)$, then $R$ is quasi-homogeneous.

Proof. For the convenience, denote $Q=k[[Y, Z]]$ and $S=Q / g Q \cong$ $P /(X, g) P$. We also write $J^{\prime}=\left(f, f_{X}, f_{Y}, f_{Z}\right) P=\left(X^{n-1}, g, g_{Y}, g_{Z}\right) P, J_{g}=\left(g_{Y}, g_{Z}\right) S$ and $\nabla g=\left(g_{Y}, g_{Z}\right) \in S^{2}$.

We prove the above only in the case $f \in \mathrm{m}_{P}^{3}$ (i.e. $n \geq 3$ and $g \in(X, Y)^{3} Q$ ), 
and leave the other (easier) cases to the reader.

Notice that $R$ is quasi-homogeneous if and only if $S$ is. Thus, in the rest, we assume that $S$ is not quasi-homogeneous (i.e. $g \notin\left(g_{Y}, g_{Z}\right) Q$ ), and we would like to derive a contradiction.

First we note that $R / J=P / J^{\prime} \cong\left(S / J_{g}\right)[X] /\left(X^{n-1}\right)$, hence that $R / J$ is a flat $S / J_{g}$-algebra.

Secondly there is an isomorphism of Koszul homologies:

$$
H_{1}(\nabla f ; R) \cong H_{1}(\nabla g ; S) \otimes_{S / J_{g}} R / J
$$

In fact, we have exact sequences which are the same as the sequence in the proof of (3.15):

$$
\begin{gathered}
0 \longrightarrow H_{1}(\nabla f ; R) \longrightarrow P /\left(X^{n-1}, g_{Y}, g_{Z}\right) P \stackrel{g}{\longrightarrow} P /\left(X^{n-1}, g_{Y}, g_{Z}\right) P \\
0 \longrightarrow H_{1}(\nabla g ; S) \longrightarrow Q /\left(g_{Y}, g_{Z}\right) Q \stackrel{g}{\longrightarrow} Q /\left(g_{Y}, g_{Z}\right) Q
\end{gathered}
$$

Noting here that $P /\left(X^{n-1}, g_{Y}, g_{Z}\right) P \cong\left(Q /\left(g_{Y}, g_{Z}\right) Q\right)[X] /\left(X^{n-1}\right)$, we obtain $H_{1}(\nabla f ; R)$ $\cong H_{1}(\nabla g ; S)[X] /\left(X^{n-1}\right)$ and this shows (4.1.1).

Thirdly we show that $H_{1}(\nabla f)=H_{1}(\nabla f ; R)$ is generated by two elements. For, since $\left(g, g_{Y}, g_{Z}\right) Q$ is an ideal of $Q$ of height two, we have its free resolution as follows:

$$
0 \longrightarrow Q^{2} \stackrel{\left(\begin{array}{ccc}
\alpha & a_{1} & a_{2} \\
\alpha^{\prime} & a_{1}^{\prime} & a_{2}^{\prime}
\end{array}\right)}{\longrightarrow} Q^{3} \stackrel{\left(\begin{array}{l}
g \\
g_{Y} \\
g_{Z}
\end{array}\right)}{\longrightarrow} Q \longrightarrow Q /\left(g, g_{Y}, g_{Z}\right) Q \longrightarrow 0 .
$$

Note that all the entries of the matrices in the sequence are non-units, since we have assumed that $g \notin\left(g_{Y}, g_{Z}\right) Q$ hence that $\left(g, g_{Y}, g_{Z}\right) Q$ is minimally generated by those three elements. See (3.1.2). It is easy to see from this sequence that $H_{1}(\nabla g ; S)$ is generated by the classes of $\left(a_{1}, a_{2}\right)$ and $\left(a_{1}^{\prime}, a_{2}^{\prime}\right) \in S^{2}$. And carefully looking at the isomorphism (4.1.1), one can show that $H_{1}(\nabla f)$ is generated by the classes of $\left(-\alpha X, a_{1}, a_{2}\right)$ and $\left(-\alpha^{\prime} X, a_{1}^{\prime}, a_{2}^{\prime}\right)$.

Finally we shall show a contradiction. Since the condition $(A)$ is assumed to hold, we can take $a \in F$ with the conditions in (3.4). From Theorem (3.12) we show that $a$ represents one of minimal generators of $H_{1}(\nabla f)$. Thus by the above, we can take $\left(-\alpha X, a_{1}, a_{2}\right)+\boldsymbol{k}$ as $\boldsymbol{a}$, where $\alpha, a_{1}, a_{2} \in(Y, Z) Q$ and $k \in K(\nabla f)$. 
The equality in (3.13) together with the above claim implies that $\mu(\mathrm{m} / \mathfrak{a})=\mu\left(H_{1}(\nabla f)\right)-1 \leq 1$ for this $a$. Further this shows that

$$
\operatorname{dim}_{k}\left(a+m_{P}^{2} / m_{P}^{2}\right) \geq 2 .
$$

Note that $-\alpha X$ and any components of $\boldsymbol{k}$ are in $\mathrm{m}^{2}$, since we have assumed that $f \in \mathrm{m}_{P}^{3}$. Thus (4.1.3) shows that $a_{1}, a_{2}$ are linearly independent as elements of $m_{P} / m_{P}^{2}$. But $a_{i} \in Q$, and we can claim from this that $\left\{a_{1}, a_{2}\right\}$ generates $(Y, Z) Q$. Therefore from (4.1.2), we see that $a_{1} \frac{\partial}{\partial Y}+a_{2} \frac{\partial}{\partial Z}$ gives a derivation on $S=Q / g Q$ and its image is just the maximal ideal. Hence it follows from a theorem of Scheja and Wiebe [11] that $S$ is quasi-homogeneous. That is against the first assumption.

Example (4.2). Suppose $f$ is of the form $X^{p}+Y^{q}+Z^{r}+X Y Z$ with $p \geq q \geq r \geq 3$. Then if the condition $(A)$ is satisfied, $R$ is quasi-homogeneous.

Proof. It is known that $R$ is always a normal domain. While we can see from an easy computation that $R$ is quasi-homogeneous (i.e. $f \in J_{P}$ $\left.=\left(f_{X}, f_{Y}, f_{Z}\right) P\right)$ if and only if $\frac{1}{p}+\frac{1}{q}+\frac{1}{r} \geq 1$. Furthermore we can verify that $\mathrm{m}_{P} f \subseteq J_{P}$ for any choice of $p, q$ and $r$. Therefore the Koszul homology $H_{1}(\nabla f)$ is given as follows:

$$
H_{1}(\nabla f) \cong J_{P}:_{P} f / J_{P}= \begin{cases}R / J & \text { if } f \in J_{P} \\ \mathrm{~m} / J & \text { if } f \notin J_{P}\end{cases}
$$

Note from the assumption that $J \subseteq \mathrm{m}^{2}$. Therefore whenever $f \notin J_{P}$, we have that $\mu\left(H_{1}(\nabla f)\right)=3$. Applying Theorem (3.15), we have only to show that $\left(J_{P}:{ }_{P} f\right) f \subseteq \mathrm{m}_{P}^{2} J_{P}$ when $R$ is not quasi-homogeneous. But in this case, we have $J_{P}:_{P} f=\mathrm{m}_{P}$ as remarked above. Thus it is enough to show that all of $X f, Y f$ and $Z f$ belong to $m_{P}^{2} J_{P}$. But this last is easily checked by some computation.

Example (4.3). Let $f$ be of the form $h+H(h)$ where $h=X^{p}+Y^{q}+Z^{r}$ with $p \geq q \geq r \geq 3$ and $H(h)$ denotes the Hessian of $h$. Then if the condition $(A)$ is satisfied, $R$ is quasi-homogeneous. 
Proof. As in the previous example, it is known and easy to see that $R$ is always a normal domain and that $R$ is quasi-homogeneous if and only if $\frac{1}{p}+\frac{1}{q}+\frac{1}{r} \geq 1$. Furthermore we can also prove that $J_{P}:_{P} f \supseteq \mathfrak{m}_{P}$ and the same equality as (4.2.1) holds. Therefore if $R$ is not quasi-homogeneous, then $J_{P}:_{P} f=\mathrm{m}_{P}$ and $\mu\left(H_{1}(\nabla f)\right)=3$. Thus to apply Theorem (3.15), it suffices to show that $f m_{P} \subseteq m_{P}^{2} J_{P}$. But an easy computation shows that each of $X f$, $Y f$ and $Z f$ is indeed in $m_{P}^{2} J_{P}$.

\section{References}

[1] Behnke, K., On Auslander modules of normal surface singularities, Manuscripta Math., 66 (1989), 205-223.

[2] Bruns, W., The Buchsbaum-Eisenbud structure theorem and alternating syzygies, Comm. Alg., 15 (1987), 873-925.

[3] Buchsbaum, D. and Eisenbud, D., What makes a complex exact?, J. Alg., 25 (1973), 259-268.

[4] Gulliksen, T.H. and Levin, G., Homology of local rings, Queen's Papers in Pure and Appl. Math. 20, Queen's Univ., Kingston, 1969.

[ 5 ] Herzog, J., On two-dimensional quasihomogeneous isolated singularities II, Arch. Math., 59 (1992), 556-561.

[6] Martsinkovsky, A., Almost split sequences and Zariski differentials, Trans. Amer. Math. Soc., 319 (1990), 285-307.

[ 7 ] , On two-dimensional quasihomogeneous isolated singularities I, Arch. Math., 59 (1992), 550-555.

[ 8 ] Saito, K., Quasihomogen isolierte Singlaritäten von Hyperfläschen, Invent. Math., 14 (1971), 123-142.

[9] Scheja, G. and Storch, U., Differentielle Eigenschaften der Lokalisierungen analytischer Algebren, Math. Ann., 197 (1972), 137-170.

[10] $\longrightarrow$ Über Spurfunktionen bei vollständigen Durchschnitten, J. Reine Angew. Math., 278/279 (1975), 174-190.

[11] Scheja, G. and Wiebe, H., Über Derivationen von Lokalen analytische Algebren, Sympos. Math., 11 (1973), 161-192.

[12] Yoshino, Y., On the structure theorem for free resolutions, Proceedings of the 7th Symposium for commutative ring theory in Japan, (1985), 195-200.

[13] - Cohen-Macaulay modules over Cohen-Macaulay rings, Lecture Note series 146, Cambridge University Press, 1990.

[14] Yoshino, Y. and Kawamoto, T., The fundamental module of a normal local domain of dimension two, Trans. Amer. Math. Soc., 309 (1988), 425-431. 